\title{
Globalizing BERT-based Transformer Architectures for Long Document Summarization
}

\author{
Quentin Grail \\ NAVER LABS Europe, \\ Meylan, France \\ Julien Perez \\ NAVER LABS Europe, \\ Meylan, France \\ Univ. Grenoble Alpes, CNRS, LIG, \\ Grenoble, France \\ quentin.grailenaverlabs.com
}

\author{
Eric Gaussier \\ Univ. Grenoble Alpes, CNRS, LIG, \\ Grenoble, France \\ eric.gaussierdimag.fr
}

\begin{abstract}
Fine-tuning a large language model on downstream tasks has become a commonly adopted process in the Natural Language Processing (NLP) (Wang et al., 2019). However, such a process, when associated with the current transformer-based (Vaswani et al., 2017) architectures, shows several limitations when the target task requires to reason with long documents. In this work, we introduce a novel hierarchical propagation layer that spreads information between multiple transformer windows. We adopt a hierarchical approach where the input is divided in multiple blocks independently processed by the scaled dot-attentions and combined between the successive layers. We validate the effectiveness of our approach on three extractive summarization corpora of long scientific papers and news articles. We compare our approach to standard and pre-trained language-model-based summarizers and report state-of-the-art results for long document summarization and comparable results for smaller document summarization.
\end{abstract}

\section{Introduction}

Language model pre-training has become a key component to improve performances on a majority of Natural Language Processing (NLP) tasks (Wang et al., 2019). Most of the recent competitive architectures (Devlin et al., 2019; Lan et al., 2020; Liu et al., 2019b; Radford et al., 2018) are based on the efficient transformer layer introduced in Vaswani et al. (2017). BERT (Devlin et al., 2019 ) is one of these architectures that has been widely adopted for comprehension and generation tasks. It is a multi-layer transformer network, pretrained with different self-supervised objectives. Numerous variations of transformer architectures have been proposed to improve this approach (Lan et al., 2020; Liu et al., 2019b; Radford et al., 2018). However, this type of process is only evaluated on tasks composed of relatively short input text, GLUE (Wang et al., 2019), SQUAD (Rajpurkar et al., 2016), SWAG (Zellers et al., 2018). Indeed, for the tasks that require reasoning with longer documents, this approach exhibits several limitations. The transformer self-attention memory quadratically increases with the number of input tokens, making it technically impossible to compute on document-scale sequences. In addition, they usually require to define a fixed maximum input length, typically of 512 tokens, at the pre-training stage.

One solution is to pre-train the entire model on longer sequences. However, this will still require a massive computation power and will only push the length limitation further. Other alternatives have been proposed to extend multi-layer transformers architectures to longer sequences without modifying this maximum length limitation. The first one is to limit the input sequence to its first tokens by removing the text beyond the length limit. Obviously, it cannot be a reasonable solution to treat long documents that are consistently longer than this limit. The second alternative is to apply the model on a window that slides all over the document. It has been used in Wolf et al. (2019) to deal with SQUAD documents that are longer than the 512 token limitation and in Joshi et al. (2019) for a co-reference resolution task on long documents. This approach can only work if the tokens need to be contextualized only in their surroundings because there is no interaction between the different windows. It seems to be a solution for co-reference resolution (Joshi et al., 2019) as they usually can be solved with a reasonably sized window. Another 
approach adopted to deal with long documents or multi-document is to select a sub-sample of the input that is small enough for the transformer model. Most of the state-of-the-art pipelines on the multihop question answering dataset HotpotQA (Yang et al., 2018) use a first model to retrieve the relevant pieces of text before feeding them to a transformerbased architecture (Fang et al., 2019a; Tu et al., 2019).

We argue that these solutions are not feasible to deal with tasks that require a global understanding of long documents. An example is extractive summarization, where the decision for each sentence should be based on the information of the complete document. To address these challenges, we propose a simple adaptation of the multi-layer transformer architecture that can scale to long documents and benefit from pre-trained parameters with a relatively small length limitation. The general idea is to independently apply a transformer network on small blocks of a text, instead of a long sequence, and to share information among the blocks between two successive layers. To the best of our knowledge, this is the first attempt to introduce hierarchical components directly between the layers of a pre-trained model and not only on top of it (Fang et al., 2019b; Zhang et al., 2019b; Tu et al., 2020). Between each of the transformer layers, we use a Bidirectional Gated Recurrent Unit (BiGRU) network (Cho et al., 2014) to spread global information across the blocks. Adding these propagation layers between the transformer layers preserves the original structure of the pre-trained model and makes it possible to transfer parameter weights from a large pre-trained language model with only few additional parameters to propagate information between blocks.

The contributions of this paper can be summarized as follows: (i) we propose a novel architecture dedicated to long documents which interweaves recurrent hierarchical modules with transformer layers and which exploits pre-trained language models like BERT, and (ii) we demonstrate that this architecture is able to build informative representations in the context of extractive summarization.

\section{Global BERT-based Transformer Architecture}

In this part, we briefly recall the transformer layer from Vaswani et al. (2017) and its integration in the BERT model (Devlin et al., 2019). Then we describe our modifications of this architecture that allow the model to read longer documents.

Transformers: The transformer architecture, based on a sequence of transformer layers, has been initially introduced in Vaswani et al. (2017). The key idea of this layer is to produce a contextualized representation of an input sequence of tokens. It is composed of the succession of a multi-head selfattention, a first normalizer, a feed-forward neural network, and a second normalizer. This model, which has originally been introduced for machine translation, has then been adopted for most natural language comprehension tasks. Most of the successful approaches (Devlin et al., 2019; Liu et al., 2019b; Lan et al., 2020) are composed of multiple stacked transformer layers. In the remainder, we denote by $T^{\ell}$ the transformation corresponding to the $\ell^{t h}, 1 \leq \ell \leq L$, transformer layer $\left(T^{\ell}\right.$ is a function from $\mathbb{R}^{N \times h}$ to $\mathbb{R}^{N \times h}$, where $N$ denotes the length of the sequence and $h$ the hidden dimension).

BERT (Devlin et al., 2019) is a multi-layer transformer encoder pre-trained on large text corpora. Two BERT architectures have been proposed in Devlin et al. (2019): BERT BASE $_{\text {composed of }}$ 12 stacked transformer layers with hidden dimension of $768(L=12, h=768)$ and BERT LARGE $_{\text {L }}$ composed of 24 layers of hidden dimension 1024 ( $L=24, h=1024)$. For both architectures, the input length is limited to 512 WordPiece tokens and the pre-training includes two self-supervised tasks, namely masked language modeling and next sentence prediction. For masked language modeling, $15 \%$ of all the WordPiece tokens of the input sequence are masked or corrupted, and the model is used to predict the original token with a crossentropy loss. For next sentence prediction, the model is trained as a classifier to predict if two sentences are contiguous or not. The pre-training procedure uses the BooksCorpus (Zhu et al., 2015) and documents from English Wikipedia. It requires 4 days of optimization on 16 TPU chips for $\mathrm{BERT}_{\mathrm{BASE}}$ and $64 \mathrm{TPU}$ chips for $\mathrm{BERT}_{\mathrm{LARGE}}$.

\subsection{Stacked Propagation Layers}

We propose a hierarchical structure that uses pretrained transformers to encode local text blocks that will be used to compute document level representations. The novel contribution of this work, depicted in Figure 1, is to incorporate recurrent hierarchical modules between the different transformer layers and not only on top of the model, as proposed in 


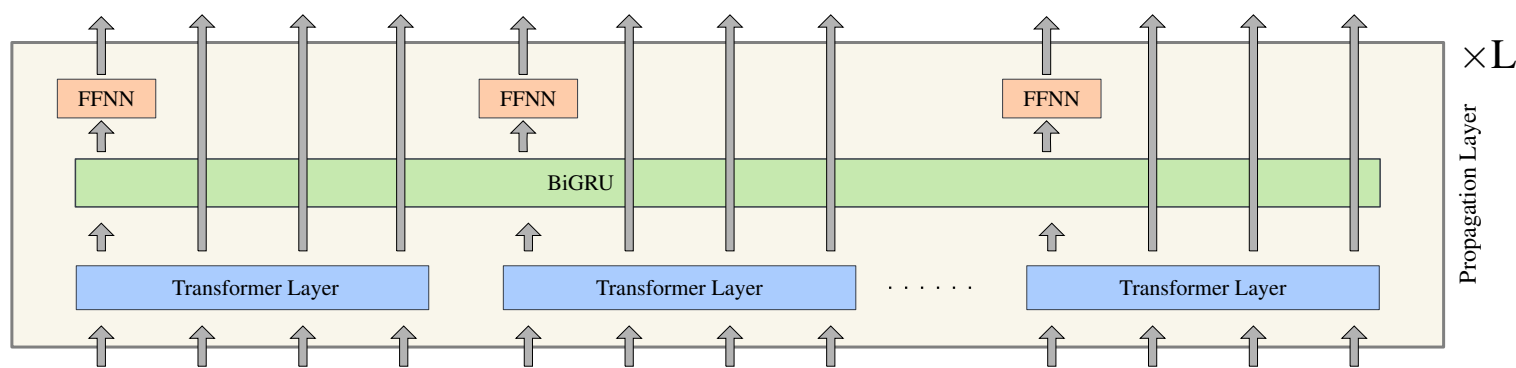

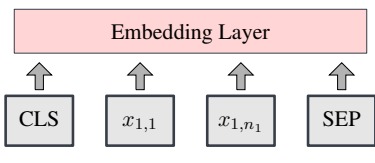

Block 1

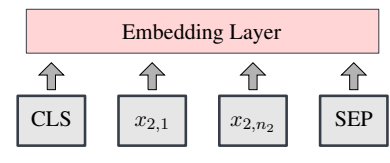

Block 2

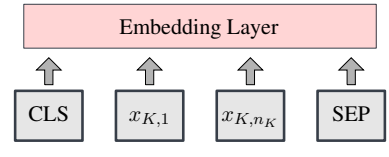

Block K

Figure 1: Our proposed modification of a multi-layer transformer architecture. The input sequence is composed of $\mathrm{K}$ blocks of tokens. Each transformer layer is applied within the blocks, and a bidirectional GRU network propagates information in the whole document by updating the [CLS] representation of each block.

several recent works (Fang et al., 2019b; Zhang et al., 2019b; Tu et al., 2020). Because we construct and propagate document level information between the layers, global and local information are fused at every level of the architecture. The text blocks can be sentences, paragraphs, or sections. We experiment using sentences as blocks because it generally does not exceed the maximum length allowed by pre-trained models and because BERT has demonstrated to be well adapted to represent such sequences.

We start by splitting the original sequence into multiple blocks. Let $D$ be a document composed of $K$ blocks, $D=\left\{B_{1} ; B_{2} ; \cdots ; B_{K}\right\}$ where a block $B_{k}, 1 \leq k \leq K$, is composed of $n_{k}$ tokens. To follow the convention of BERT, special tokens [CLS] and $[\mathrm{SEP}]$ are respectively added at the beginning and end of each block of the document, so that: $B_{k}=\left\{[\mathrm{CLS}] ; x_{k, 1} ; x_{k, 2} ; \cdots x_{k, n_{k}} ;[\mathrm{SEP}]\right\}$ where $x_{k, i}$ is the index of the WordPiece token $i$ of block $k$. In the remainder, the index 0 (resp. $n_{k}+1$ ) will be used to refer to the representation of the [CLS] (resp. [SEP]) token in each block.

Embedding Layer Because our goal is to reuse the available pre-trained BERT parameters, token representations are kept the same as in the original BERT and are composed of a token embedding, a segment embedding, and a positional encoding that represents the position of the token in its block. We will denote by $E_{k}\left(E_{k} \in \mathbb{R}^{\left(n_{k}+2\right) \times h}, 1 \leq k \leq K\right)$ the embedding representation of block $k$.

Propagation Layers Our model is composed of $L$ stacked identical hierarchical layers, called propagation layers, that comprise a transformer layer, a BiGRU to propagate information across blocks and, finally, a feed-forward network. For any layer $\ell, 1 \leq \ell \leq L$, let $U_{k}^{\ell} \in \mathbb{R}^{\left(n_{k}+2\right) \times h}$ be the representation of block $k$ after the $(\ell-1)^{t h}$ layer, the representation for the first layer being initialized with the output of the embedding layer: $U_{k}^{1}=E_{k}, \quad \forall k \in\{1, \cdots, K\}$. We first apply the pre-trained transformer function $T^{\ell}$ individually on each block of the document to compute local, token-aware representations $V_{k}^{\ell} \in \mathbb{R}^{\left(n_{k}+2\right) \times h}$ :

$$
V_{k}^{\ell}=T^{\ell}\left(U_{k}^{\ell}\right), \quad \forall k \in\{1, \cdots, K\} .
$$

The next step is to propagate information across all the blocks of the document in order to compute a global block-aware representation for the document at layer $\ell$, denoted by $W^{\ell} \in \mathbb{R}^{K \times h}, 1 \leq k \leq K$. To do so, we use a BiGRU network, fed with the representation vectors of the different blocks, and apply a feed-forward neural network to preserve the hidden dimension of the transformer. Each block $k$ is represented by its [CLS] vector, i.e., the vector (represented by $V_{k, 0}^{\ell} \in \mathbb{R}^{h}$ ) at the first position in the local representation of the block. These representations are then concatenated to form the input to the BiGRU. The global, block-aware representation is then computed by applying the feed-forward neural network (FFNN) to all $K$ outputs of the BiGRU:

$$
W_{k}^{\ell}=\operatorname{FFNN}\left(\operatorname{BiGRU}_{k}\left(\left[V_{1,0}^{\ell} ; \cdots ; V_{K, 0}^{\ell}\right]\right)\right),
$$




\begin{tabular}{l|cc|cc}
\hline \multirow{2}{*}{ Datasets } & \multicolumn{2}{|c}{ avg. doc length } & \multicolumn{2}{c}{ avg. summary length } \\
& sentences & words & sentences & words \\
\hline arXiv & 204 & 5038 & 5.6 & 165 \\
PubMed & 88 & 3235 & 6.8 & 205 \\
CNN/DM & 32 & 757 & 4.1 & 57 \\
\hline
\end{tabular}

Table 1: Statistics on arXiv, PubMed and CNN/DailyMail validation datasets in terms of documents and summary lengths.

where $\mathrm{BiGRU}_{k}$ denotes the $k^{\text {th }}$ output of the $\mathrm{Bi}$ GRU and [;] is the concatenation operation.

At this stage, we have computed, for a given document, local block representations $V_{k}^{\ell}(1 \leq k \leq$ $K)$ and a global representation $W^{\ell}$. We combine them to build the output representation of the layer:

$$
U_{k}^{\ell+1}=\left[W_{k}^{\ell} ; V_{k, 1}^{\ell} ; \cdots ; V_{k, n_{k}+1}^{\ell}\right], \quad 1 \leq k \leq K .
$$

As one can note, $U_{k}^{\ell+1} \in \mathbb{R}^{\left(n_{k}+2\right) \times h}$ is a representation of block $k$ in which the [CLS] vector representation has been enriched with document level information propagated from other blocks. $U_{k}^{\ell+1}$ is then used as input for the next propagation layer.

\subsection{Output Layer}

In this work, we validate our approach on the task of extractive summarization described in Section 3. This task can be considered as a binary classification problem where each block has to be labeled as selected or not. We use a feed-forward neural network followed by a Softmax function on the top of the block level representations after the last layer $L$ to compute $Y \in \mathbb{R}^{K \times 2}$.

$$
Y_{k}=\operatorname{Softmax}\left(\operatorname{FFNN}\left(W_{k}^{L+1}\right)\right) .
$$

Using a recurrent architecture to propagate information between blocks has two interesting properties. First, it allows our model to scale to long sequences of blocks without using an attention mechanism that would not scale. Second, it does not require to implement any positional encoding on block representations.

\section{Experiments}

We evaluate our approach, which we refer to as GBT-EXTSUM (for 'Global BERT-based Transformer for Extractive Summarization'), in the context of extractive summarization, the goal of which being to identify and extract from a document the pieces of text that are the most important (Kupiec et al., 1995). We view this task as a sentencelevel classification problem where each sentence has to be labeled according to its belonging to the summary or not. To validate the effectiveness of our approach, we propose to test it on three summarization datasets, namely ArXiv, PubMed and CNN/DailyMail:

- The ArXiv and Pubmed datasets have been introduced in Cohan et al. (2018). They contain long scientific documents from arXiv. org and Pubmed.com and use their abstracts as the ground-truth summaries. We use the original splits that respectively contain $203,037 / 6,436 / 6,440$ samples in the training, validation, and test sets for arXiv, and 119,924/6,633/6,658 for PubMed.

- The CNN/DailyMail dataset contains news articles associated with short summaries. We use the splits of Hermann et al. (2015), where entities have not been anonymized. This dataset contains 287,226 training samples, 13,368 validation samples, and 11,490 test samples.

Table 1 presents some statistics on these three datasets. As one can note, for the scientific articles, the average number of tokens in the documents to summarize is way beyond the capabilities of a standard transformer pre-trained with BERT.

\subsection{Evaluation Metrics}

We evaluate the quality of the extracted summaries using the ROUGE metric (Lin, 2004), and more particularly ROUGE-1 (overlap of unigrams), ROUGE-2 (overlap of bigrams), ROUGE-3 (overlap of trigrams) and ROUGE-L (longest common subsequence between the produced summary and the gold-standard one).

\subsection{Label Generation}

In order to train extractive summarizers, one needs annotations in the form of sentence-level binary labels. To compute such annotations, we follow the work of Kedzie et al. (2018) and label all sentences by greedily optimizing the ROUGE-1 score of the extracted summary against the gold-standard summary associated with each article. These labels are only used at training time, the evaluation of the extracted summaries being done against the gold-standard summaries provided in the datasets. 


\begin{tabular}{|c|c|c|c|c|c|c|c|c|c|}
\hline \multirow{2}{*}{\multicolumn{2}{|c|}{ Summarizer }} & \multicolumn{4}{|c|}{ PubMed } & \multicolumn{4}{|c|}{ arXiv } \\
\hline & & RG-1 & RG-2 & RG-3 & RG-L & RG-1 & RG-2 & RG-3 & RG-L \\
\hline \multirow{9}{*}{ 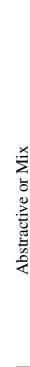 } & Oracle & 58.15 & 34.16 & 24.11 & 52.99 & 57.78 & 30.43 & 18.41 & 51.24 \\
\hline & Lead & 37.77 & 13.35 & 7.64 & 34.31 & 35.54 & 9.50 & 3.33 & 31.19 \\
\hline & Attn-Seq2Seq (Nallapati et al., 2016) & 31.55 & 8.52 & 7.05 & 27.38 & 29.30 & 6.00 & 1.77 & 25.56 \\
\hline & Pntr-Gen-Seq2Seq (See et al.) & 35.86 & 10.22 & 7.60 & 29.69 & 32.06 & 9.04 & 2.15 & 25.16 \\
\hline & Discourse summarizer (Cohan et al., 2018) & 38.93 & 15.37 & 9.97 & 35.21 & 35.80 & 11.05 & 3.62 & 31.80 \\
\hline & TLM-I+E (G,M) (Subramanian et al., 2019) & 42.13 & 16.27 & 8.82 & 39.21 & 41.62 & 14.69 & 6.16 & 38.03 \\
\hline & DANCER PEGASUS (Gidiotis and Tsoumakas, 2020) & 46.34 & 19.97 & - & 42.42 & 45.01 & 17.60 & - & 40.56 \\
\hline & PEGASUS (Zhang et al., 2019a) & 45.97 & 20.15 & - & 28.25 & 44.21 & 16.95 & - & 25.67 \\
\hline & BIGBIRD-Pegasus (Zaheer et al., 2020) & 46.32 & 20.65 & - & 42.33 & 46.63 & 19.02 & - & 41.77 \\
\hline \multirow{11}{*}{ 总 } & SumBasic (Vanderwende et al., 2007) & 37.15 & 11.36 & 5.42 & 33.43 & 29.47 & 6.95 & 2.36 & 26.30 \\
\hline & LexRank (Erkan and Radev, 2004) & 39.19 & 13.89 & 7.27 & 34.59 & 33.85 & 10.73 & 4.54 & 28.99 \\
\hline & LSA (Steinberger and Jezek, 2004) & 33.89 & 9.93 & 5.04 & 29.70 & 29.91 & 7.42 & 3.12 & 25.67 \\
\hline & Sent-CLF (Subramanian et al., 2019) & 45.01 & 19.91 & 12.13 & 41.16 & 34.01 & 8.71 & 2.99 & 30.41 \\
\hline & Sent-PTR (Subramanian et al., 2019) & 43.30 & 17.92 & 10.67 & 39.47 & 42.32 & 15.63 & 7.49 & 38.06 \\
\hline & Bert Ranker (Nogueira and Cho, 2019) & 43.67 & 18.00 & 10.74 & 39.22 & 41.65 & 13.88 & 5.92 & 36.40 \\
\hline & BERTSUMEXT (Liu and Lapata, 2019b) & 41.09 & 15.51 & 8.64 & 36.85 & 41.24 & 13.01 & 5.26 & 36.10 \\
\hline & BERTSUMEXT (SW) (Liu and Lapata, 2019b) & 45.01 & 20.00 & 12.05 & 40.43 & 42.93 & 15.08 & 6.01 & 37.22 \\
\hline & Longformer-Ext (Beltagy et al., 2020) & 43.75 & 17.37 & 10.18 & 39.71 & 45.24 & 16.88 & 8.06 & 40.03 \\
\hline & Reformer-Ext (Kitaev et al., 2020) & 42.32 & 15.91 & 9.02 & 38.26 & 43.26 & 14.86 & 6.66 & 38.10 \\
\hline & GBT-ExTSUm (Ours) & 46.87 & 20.19 & 12.11 & 42.68 & 48.08 & 19.21 & 9.58 & 42.68 \\
\hline
\end{tabular}

Table 2: Summarization results on PubMed and arXiv. Except for BERT-based approaches, for Reformer-Ext and for Longformer-Ext, which we have reimplemented, the results of the baselines are taken from their associated paper as well as from Cohan et al. (2018). Bold results correspond to the best scores of extractive summarizers.

\subsection{Baseline Models}

We compare our approach to several well known published methods described below. These methods include SumBasic (Vanderwende et al., 2007), LexRank (Erkan and Radev, 2004), LSA (Steinberger and Jezek, 2004), Attn-Seq2Seq (Nallapati et al., 2016), Pntr-Gen-Seq2Seq (See et al.) and Discourse-aware summarizer (Cohan et al., 2018). The results for these models are the ones reported in the paper (Cohan et al., 2018). We also report the results of Sent-CLF and Sent-PTR, which are hierarchical sentence pointer and classifier, TLM$\mathrm{I}+\mathrm{E}(\mathrm{G}, \mathrm{M})$ a mixed extractive/generative transformer language model from Subramanian et al. (2019), BIGBIRD (Zaheer et al., 2020), PEGASUS (Zhang et al., 2019a) and DANCER (Gidiotis and Tsoumakas, 2020) which are three abstractive methods. Lastly, we developed several baseline models based on BERT, Longformer (Beltagy et al., 2020) and Reformer (Kitaev et al., 2020):

BERT Ranker: We used a BERT ranker, similar to Nogueira and Cho (2019) in which each sentence of the document is processed individually. We apply BERT on each sentence ${ }^{1}$ and use a Sigmoid layer, the input of which consists of the

\footnotetext{
${ }^{1}$ This is possible as no sentence exceeds BERT token limi-
}

[CLS] representation of the sentence, to model the probability of the sentence to be selected.

BERTSUMEXT has been introduced in Liu and Lapata (2019b). This model is an adaptation of BERT for extractive summarization. Because this model takes as input the concatenation of all the tokens of the document, it cannot scale to the arXiv and PubMed datasets. We propose two variants: the first one is to take as input only the first 800 tokens of the document, as suggested in the original paper. This solution is displayed as BERTSUMEXT in Table 2. The second is to apply BERTSUMEXT per sliding windows on the original document and to use, as a token representation, its representation in the window that maximizes its surrounding context. We name this sliding window implementation BERTSUMEXT (SW) in Table 2.

Longformer-Ext and Reformer-Ext: The Longformer and Reformer models were respectively introduced by Beltagy et al. (2020) and Kitaev et al. (2020). They both propose an adaptation of the Transformer self-attention that scale to long sequences. We add the same classification head as the one used in our model on top of the contextualized representation of the first token of each sentence to label them as selected or not in the summary. 
We also present the Oracle extractive results as an upper bound as well as the Lead baseline (which respectively select the first 3, 6, 7 sentences for CNN/DailyMail, arXiv and PubMed datasets). Several models are reported only on CNN/DailyMail dataset and not on arXiv/Pubmed as they do not scale to long documents.

\subsection{Implementation details}

We run all our experiments using the Pytorch library (Paszke et al., 2019). We built our model using the "bert-base-uncase" ${ }^{2}$ version of BERT and its implementation in the HuggingFace library (Wolf et al., 2019). Our architecture is composed of $L=12$ propagation layers with a transformer hidden dimension of $h=768$. The hidden dimension of the BiGRU is set to 384 and we share its parameters among all the propagation layers. The FFNN inside the propagation layers maps the output of the BiGRU of dimension $2 \times 384$ to a vector of dimension 768 . The FFNN of the output layer is a binary classifier that projects the sentence representations of dimension 768 to an output of dimension 2. We fine-tuned our model on the crossentropy loss, for 5 epochs on 4 GPUs V100 and use Adam optimizer (Kingma and $\mathrm{Ba}, 2015$ ) with the initial learning rate set to $3 \times 10^{-5}, \beta_{1}=0.9$, $\beta_{2}=0.999$, no learning rate warmup and a linear decay of the learning rate. We describe implementation details of BERTSUMEXT, Longformer-Ext and Reformer-Ext baselines in the Supplementary Material, Appendix A.

We used Trigram Blocking to avoid the repetition of trigrams in the extracted summaries as suggested in Paulus et al. (2018). Given the extracted summary so far, we only added candidate sentences that had no overlapping trigram with the current summary. We limited the summary to 3 sentences for the CNN/DailyMail dataset, 6 sentences for arXiv, and 7 for PubMed.

\subsection{Results}

Our main results are shown in Tables 2 and 3. On the arXiv and PubMed datasets, our model outperforms the baseline models on almost all of the reported metrics. Our approach manages to summarize long documents while preserving informativeness (evaluated by ROUGE-1) and fluency (evaluated by ROUGE-L) of the summaries. In addition

\footnotetext{
${ }^{2}$ https://github.com/google-research/ bert
}

\begin{tabular}{|c|c|c|c|}
\hline Model & $\mathrm{R}-1$ & $\mathrm{R}-2$ & $\mathrm{R}-\mathrm{L}$ \\
\hline Oracle & 56.22 & 33.74 & 52.19 \\
\hline Lead-3 & 40.11 & 17.54 & 36.32 \\
\hline LATENT (Zhang et al., 2018) & 41.05 & 18.77 & 37.54 \\
\hline NEUSUM (Zhou et al., 2018) & 41.59 & 19.01 & 37.98 \\
\hline Sumo (Liu et al., 2019a) & 41.00 & 18.40 & 37.20 \\
\hline TransformerExt (Liu and Lapata, 2019b) & 40.90 & 18.02 & 37.17 \\
\hline MASK-LM ${ }^{\text {global }}$ (Chang et al., 2019) & 41.2 & 19.1 & 37.6 \\
\hline PNBERT (Zhong et al., 2019) & 42.69 & 19.60 & 38.85 \\
\hline BERT-ext + RL (Bae et al., 2019) & 42.76 & 19.87 & 39.11 \\
\hline HIBERT $_{M}$ (Zhang et al., 2019b) & 42.37 & 19.95 & 38.83 \\
\hline BERTSUMEXT (Liu and Lapata, 2019b) & 43.25 & 20.24 & 39.63 \\
\hline BERTSUMEXT w/o interval embedding & 43.20 & 20.22 & 39.59 \\
\hline BERTSUMEXT (large) & 43.85 & 20.34 & 39.90 \\
\hline MatchSum (RoBERTa) (Zhong et al., 2020) & 44.41 & 20.86 & 40.55 \\
\hline Reformer-Ext (Kitaev et al., 2020) & 38.85 & 16.46 & 35.16 \\
\hline Longformer-Ext (Beltagy et al., 2020) & 43.00 & 20.20 & 39.30 \\
\hline GBT-ExTSum (Ours) & 42.93 & 19.81 & 39.20 \\
\hline
\end{tabular}

Table 3: Comparison of ROUGE scores on CNN/DailyMail wrt extractive models. All results are taken from original papers but Reformer-Ext and Longformer-Ext which we have reimplemented.

to the previously published methods, our approach also improves over the BERT-based, LongformerExt and Reformer-Ext baselines we have developed. Among them, BERTSUMEXT, which focuses on a truncated version of the document, is the less effective. As documents are significantly longer than the 800 tokens limitation of this model, this result is not surprising. The sliding window adaptation of this model, that allows it to scale to long documents, is the one that achieves results that are the most comparable to ours. Our approach still outperforms this adaptation, demonstrating that summaries require to propagate information beyond a single BERT window.

On the CNN/DailyMail dataset, one can see that our model outperforms all the models that do not use pre-trained parameters. This includes several transformer-based and hierarchical models. However, while having comparable results, we do not achieve stronger performance than the current extractive state of the art from Zhong et al. (2020). This is not surprising as the majority of the CNN/DailyMail examples contains their oracle summary sentences in the first positions of the articles, as shown in the Supplementary Material, Appendix B.

Lastly, we evaluate the impact of several elements of our proposed model in Table 4. We first study the influence of the underlying language model by considering both RoBERTa (Liu et al., 2019b) and PEGASUS (Zhang et al., 2019a) pre-trained models, respectively referred to as GBT-EXTSUM-RoBERTa and GBT-EXTSUM- 


\begin{tabular}{l|c|c|c|c|c|c} 
& \multicolumn{3}{|c}{ PubMed } & \multicolumn{3}{c}{ arXiv } \\
Model & R-1 & R-2 & R-L & R-1 & R-2 & R-L \\
\hline GBT-EXTSum & 46.87 & 20.19 & 42.68 & 48.08 & 19.21 & 42.68 \\
\hline \hline GBT-EXTSUM-RoBERTa & 46.02 & 19.29 & 41.84 & 47.42 & 18.62 & 42.03 \\
GBT-EXTSum-PEGASUS & 44.11 & 17.34 & 40.03 & 43.50 & 15.35 & 38.41 \\
\hline \hline GBT-EXTSuM-NoShare & 46.84 & 20.19 & 42.63 & 48.11 & 19.30 & 42.75 \\
\hline \hline GBT-EXTSUM-AveragePool & 45.24 & 18.13 & 40.94 & 45.71 & 17.36 & 40.43 \\
GBT-EXTSum-Transformer & 46.46 & 19.62 & 42.17 & 47.64 & 18.82 & 42.22 \\
\hline
\end{tabular}

Table 4: Analysis of the influence of different key components of our proposed architecture.

PEGASUS. As one can see, the results show that BERT-base architecture performs best in terms of ROUGE scores on both arXiv and PubMed. One major difference between PEGASUS and BERT/RoBERTA pre-trained models is that BERT/RoBERTA are only encoders while PEGASUS is a pre-trained encoder/decoder architecture. This could explain why BERT/RoBERTA outperform PEGASUS on extractive summarization tasks. We then compare an alternative of our implementation of GBT-EXTSUM in which the parameters of the BiGRU are not shared among all the propagation layers (GBT-EXTSUM-NoShare) and found no clear difference with the version in which the parameters are shared. Lastly, we compare three architectures of propagation layers, including an average pooling of the [CLS] representations of the sentences, a Transformer layer between the [CLS] tokens (associated to a block position embedding), and a BiGRU layer. Among these three layers, the average pooling layer, which introduces no additional trainable parameters, performs the worst. Furthermore, the BiGRU layer slightly outperforms the Transformer layer in terms of ROUGE scores.

Analysis. In Figure 2, we compare the R-1 score of several models regarding the number of words in the source documents. One can see that GBTEXTSUM consistently outperforms BERTSUMEXT (SW), Reformer-Ext and Longformer-Ext regardless of the number of words in the source documents.

We present in Table 5 two example summaries of a document from the PubMed test set (Kamio et al., 2009), respectively obtained by GBT-EXTSUM and BERTSUMEXT (SW). The numbers in the margin indicate the position of the sentences in the original document, which is composed of a total of 78 sentences. As one can observe, GBT-EXTSUM extracts sentences from various parts of the document whereas BERTSUMEXT (SW) mostly focuses

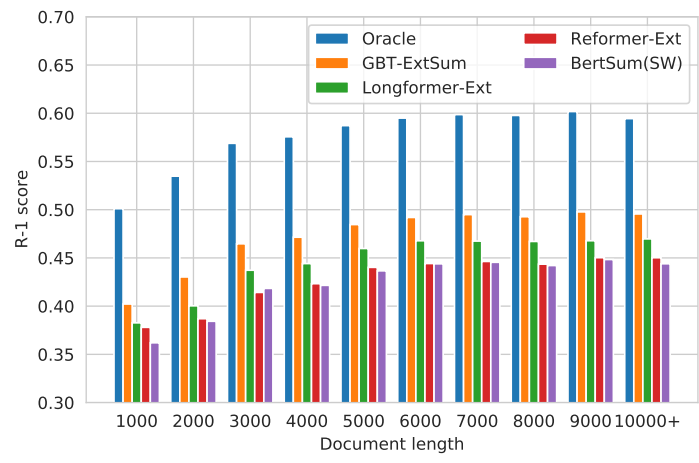

Figure 2: Average R-1 scores of extracted summaries according to the number of words in the input documents from arXiv test dataset.

on the beginning of the document. Among the sentences selected by the two models, the most meaningful one, in terms of ROUGE, is the last one selected by GBT-EXTSUM. This sentence appears at position 66, in the last section (Discussion) of the original paper. In contrast, BERTSUMEXT (SW) proposes sentences that are less relevant for summarization purposes. Additional summaries of the PubMed and arXiv articles are provided in the Supplementary Material, Appendices C and D.

To analyse the influence of the positions of the sentences in the input document, we present in Figure 3 the histograms of the positions of the sentences of the Oracle summary as well as that of the predicted positions of different models, on the PubMed test set. One can see that if most relevant sentences appear at the beginning of a document, other Oracle sentences are still relevant further down the document. GBT-EXTSUM is the model that behaves the most closely to the Oracle, followed by BERTSUMEXT (SW), Reformer-Ext and Longformer-Ext. These last two models tend to over-select sentences from the beginning while focusing less on the ones appearing later in the document. Our model remains influenced by the sentence position but is still able to select sentences from all over the document and is closer to the Oracle distribution.

\section{Related Work}

Hierarchical neural architectures have been competitive on a collection of NLP tasks that require to reason over long or multiple documents such as aspect-based sentiment analysis (Paulus et al., 2018), document summarization (Cheng and Lapata, 2016), document segmentation (Koshorek 


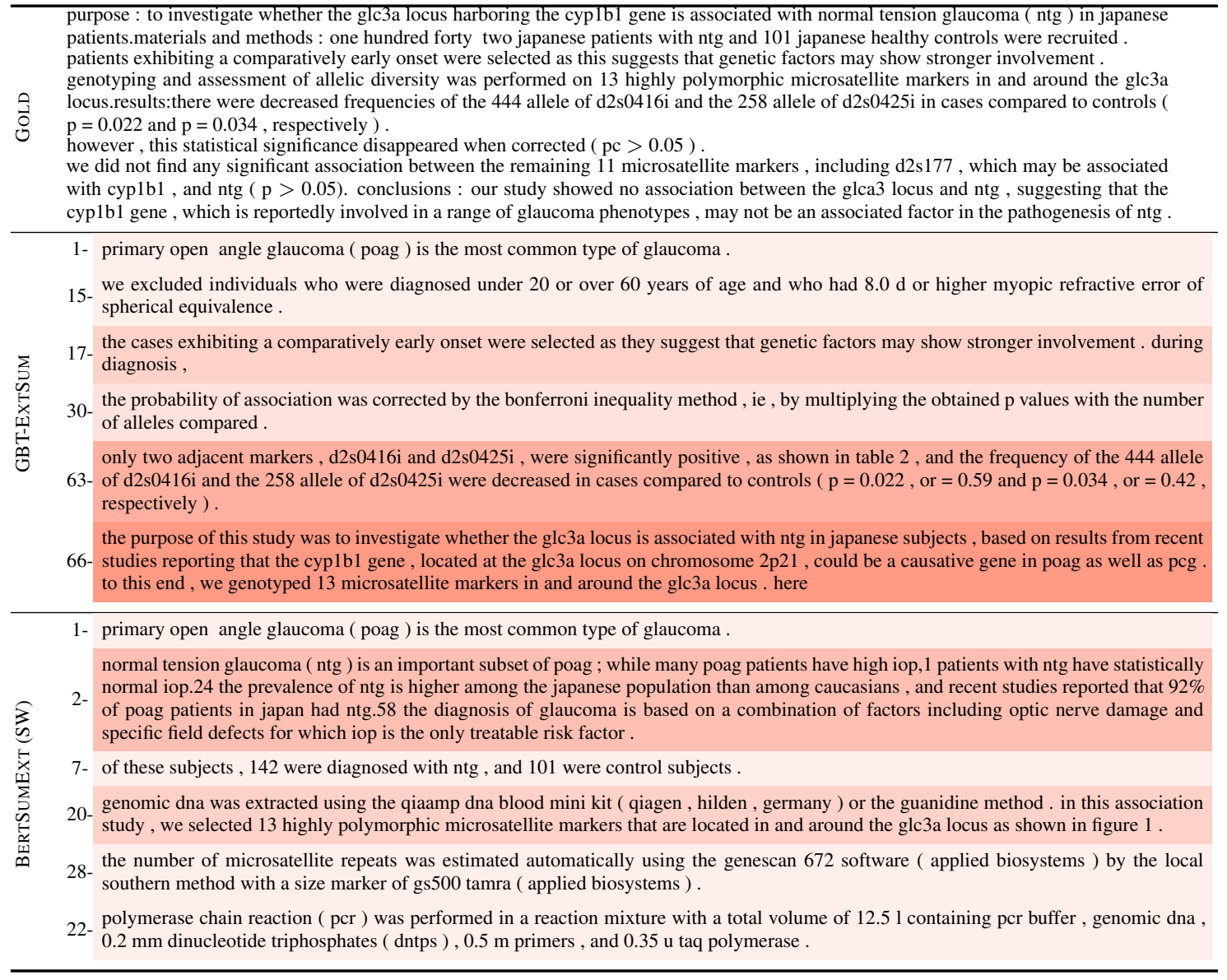

Table 5: An example of summary produced by our method compared to the gold summary and one produced by BERTSUMEXT (SW). With a red scale, we highlight the sentences with the highest ROUGE score when evaluated against the abstract. We show in the margin the position of the extracted sentence in the document. This document (Kamio et al., 2009) is 78 sentences long.

et al., 2018) and text classification (Yang et al., 2016). The hierarchical structure enables the model to learn local contextualized token representations in its lower hierarchy level, while higher-level representations can capture long-distance dependencies within the document. Liu and Lapata (2019a) have proposed a hierarchical modification of the transformer layer-based attention modules to model relations between documents for abstractive summarization but do not investigate parameter transfer form pre-trained language models. Chang et al. (2019) and Zhang et al. (2019b) suggested pretraining processes for hierarchical models, without however testing their approaches on long document summarization nor releasing their pre-trained models. We have not included these models in our comparison for this reason. Transformer-XH (Zhao et al., 2020) introduced an eXtra Hop attention to model dependencies between different transformer windows but requires a graph of related documents.

Long-Document Transformers: Multiple studies have investigated different self-attention mechanisms to extend transformers to long documents. Transformer-XL (Dai et al., 2019) introduced a recurrence between successive transformer windows which run from left to right through the document, preventing global information to bidirectionally flow through the document. Other approaches design the self-attention as a sparse layer, as sparse transformers (Child et al., 2019) or the recently proposed Longformer and BIGBIRD models (Beltagy et al., 2020; Zaheer et al., 2020). One major difference with our work is that these models compute the attention only between a limited set of randomly or a priori chosen tokens. Reformer (Kitaev et al., 2020) also tackles the problem of language modeling for long sequences, but it does so by computing the self-attention only between similar 


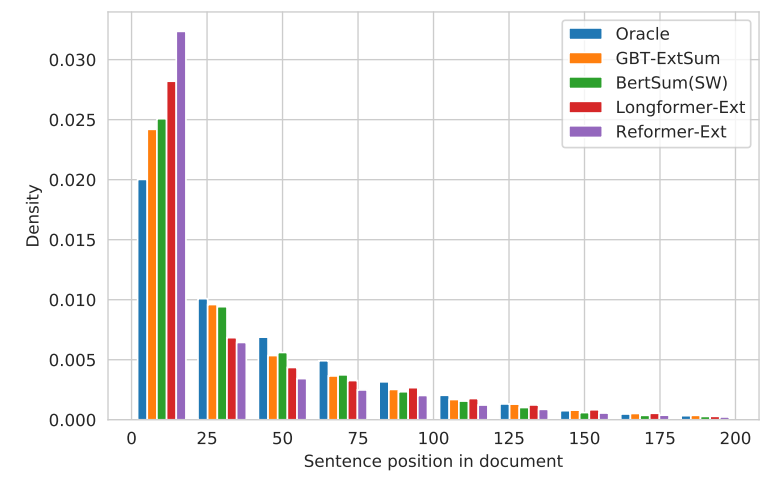

Figure 3: Proportion of the extracted sentences according to their position in the input document from PubMed test dataset.

tokens, based on locality-sensitive hashing.

\section{Conclusion}

In this paper, we have introduced a novel transformer-based model for long document summarization based on propagation layers that spread information between multiple transformer windows. This model preserves the architecture of commonly used pre-trained language models, thus allowing the transfer of parameters. An evaluation, conducted on top of the BERT model in the context of an extractive summarization task, further revealed its effectiveness in dealing with long documents compared to other adaptations of BERT and previously proposed models. In the future, we plan to adapt our model to other tasks that require understanding long documents, as question-answering and document-scale machine translation.

\section{Acknowledgments}

This work was partially supported by MIAI@Grenoble Alpes, (ANR-19-P3IA-0003).

\section{References}

Sanghwan Bae, Taeuk Kim, Jihoon Kim, and Sanggoo Lee. 2019. Summary level training of sentence rewriting for abstractive summarization. CoRR, abs/1909.08752.

Iz Beltagy, Matthew E. Peters, and Arman Cohan. 2020. Longformer: The long-document transformer. CoRR, abs/2004.05150.

Ming-Wei Chang, Kristina Toutanova, Kenton Lee, and Jacob Devlin. 2019. Language model pre-training for hierarchical document representations. CoRR, abs/1901.09128.
Jianpeng Cheng and Mirella Lapata. 2016. Neural summarization by extracting sentences and words. In Proceedings of the 54th Annual Meeting of the Association for Computational Linguistics (Volume 1: Long Papers), pages 484-494, Berlin, Germany. Association for Computational Linguistics.

Rewon Child, Scott Gray, Alec Radford, and Ilya Sutskever. 2019. Generating long sequences with sparse transformers. CoRR, abs/1904.10509.

Kyunghyun Cho, Bart van Merriënboer, Caglar Gulcehre, Dzmitry Bahdanau, Fethi Bougares, Holger Schwenk, and Yoshua Bengio. 2014. Learning phrase representations using RNN encoder-decoder for statistical machine translation. In Proceedings of the 2014 Conference on Empirical Methods in Natural Language Processing (EMNLP), pages 17241734, Doha, Qatar. Association for Computational Linguistics.

Arman Cohan, Franck Dernoncourt, Doo Soon Kim, Trung Bui, Seokhwan Kim, Walter Chang, and Nazli Goharian. 2018. A discourse-aware attention model for abstractive summarization of long documents. In Proceedings of the 2018 Conference of the North American Chapter of the Association for Computational Linguistics: Human Language Technologies, NAACL-HLT, New Orleans, Louisiana, USA, June 16, 2018, Volume 2 (Short Papers), pages 615-621. Association for Computational Linguistics.

Zihang Dai, Zhilin Yang, Yiming Yang, Jaime G. Carbonell, Quoc Viet Le, and Ruslan Salakhutdinov. 2019. Transformer-xl: Attentive language models beyond a fixed-length context. In Proceedings of the 57th Conference of the Association for Computational Linguistics, ACL 2019, Florence, Italy, July 28-August 2, 2019, Volume 1: Long Papers, pages 2978-2988. Association for Computational Linguistics.

Jacob Devlin, Ming-Wei Chang, Kenton Lee, and Kristina Toutanova. 2019. BERT: Pre-training of Deep Bidirectional Transformers for Language Understanding. In Proceedings of the 2019 Conference of the North American Chapter of the Association for Computational Linguistics: Human Language Technologies, Volume 1 (Long and Short Papers), pages 4171-4186. Association for Computational Linguistics.

Günes Erkan and Dragomir R. Radev. 2004. Lexrank: Graph-based lexical centrality as salience in text summarization. J. Artif. Int. Res., 22(1):457-479.

Yuwei Fang, Siqi Sun, Zhe Gan, Rohit Pillai, Shuohang Wang, and Jingjing Liu. 2019a. Hierarchical graph network for multi-hop question answering. CoRR, abs/1911.03631.

Yuwei Fang, Siqi Sun, Zhe Gan, Rohit Pillai, Shuohang Wang, and Jingjing Liu. 2019b. Hierarchical graph network for multi-hop question answering. CoRR, abs/1911.03631. 
Alexios Gidiotis and Grigorios Tsoumakas. 2020. A divide-and-conquer approach to the summarization of long documents.

Karl Moritz Hermann, Tomas Kocisky, Edward Grefenstette, Lasse Espeholt, Will Kay, Mustafa Suleyman, and Phil Blunsom. 2015. Teaching machines to read and comprehend. In Advances in Neural Information Processing Systems, volume 28, pages 16931701. Curran Associates, Inc.

Mandar Joshi, Omer Levy, Luke Zettlemoyer, and Daniel S. Weld. 2019. BERT for coreference resolution: Baselines and analysis. In Proceedings of the 2019 Conference on Empirical Methods in Natural Language Processing and the 9th International Joint Conference on Natural Language Processing, EMNLP-IJCNLP 2019, Hong Kong, China, November 3-7, 2019, pages 5802-5807. Association for Computational Linguistics.

M Kamio, Akira Meguro, Masao Ota, N Nomura, Kenji Kashiwagi, F Mabuchi, Hiroyuki Iijima, K Kawase, T Yamamoto, M Nakamura, Akira Negi, T Sagara, Teruo Nishida, M Inatani, Hidenobu Tanihara, M Aihara, M Araie, Takeo Fukuchi, H Abe, and Nakamura Mizuki. 2009. Investigation of the association between the glc3a locus and normal tension glaucoma in japanese patients by microsatellite analysis. Clinical ophthalmology (Auckland, N.Z.), 3:183-8.

Chris Kedzie, Kathleen McKeown, and Hal Daumé III. 2018. Content selection in deep learning models of summarization. In Proceedings of the 2018 Conference on Empirical Methods in Natural Language Processing, pages 1818-1828, Brussels, Belgium. Association for Computational Linguistics.

Diederik P. Kingma and Jimmy Ba. 2015. Adam: A method for stochastic optimization. In 3rd International Conference on Learning Representations, ICLR 2015, San Diego, CA, USA, May 7-9, 2015, Conference Track Proceedings.

Nikita Kitaev, Lukasz Kaiser, and Anselm Levskaya. 2020. Reformer: The efficient transformer. In 8th International Conference on Learning Representations, ICLR 2020, Addis Ababa, Ethiopia, April 2630, 2020. OpenReview.net.

Omri Koshorek, Adir Cohen, Noam Mor, Michael Rotman, and Jonathan Berant. 2018. Text segmentation as a supervised learning task. In Proceedings of the 2018 Conference of the North American Chapter of the Association for Computational Linguistics: $\mathrm{Hu}$ man Language Technologies, Volume 2 (Short Papers), pages 469-473, New Orleans, Louisiana. Association for Computational Linguistics.

Julian Kupiec, Jan Pedersen, and Francine Chen. 1995. A trainable document summarizer. In Proceedings of the 18th Annual International ACM SIGIR Conference on Research and Development in Information Retrieval, SIGIR '95, page 68-73, New York, NY, USA. Association for Computing Machinery.
Zhenzhong Lan, Mingda Chen, Sebastian Goodman, Kevin Gimpel, Piyush Sharma, and Radu Soricut. 2020. Albert: A lite bert for self-supervised learning of language representations. In International Conference on Learning Representations.

Chin-Yew Lin. 2004. ROUGE: A package for automatic evaluation of summaries. In Text Summarization Branches Out, pages 74-81, Barcelona, Spain. Association for Computational Linguistics.

Yang Liu and Mirella Lapata. 2019a. Hierarchical transformers for multi-document summarization. In Proceedings of the 57th Conference of the Association for Computational Linguistics, ACL 2019, Florence, Italy, July 28-August 2, 2019, Volume 1: Long Papers, pages 5070-5081. Association for Computational Linguistics.

Yang Liu and Mirella Lapata. 2019b. Text summarization with pretrained encoders. In Proceedings of the 2019 Conference on Empirical Methods in Natural Language Processing and the 9th International Joint Conference on Natural Language Processing (EMNLP-IJCNLP), pages 3730-3740, Hong Kong, China. Association for Computational Linguistics.

Yang Liu, Ivan Titov, and Mirella Lapata. 2019a. Single document summarization as tree induction. In Proceedings of the 2019 Conference of the North American Chapter of the Association for Computational Linguistics: Human Language Technologies, Volume 1 (Long and Short Papers), pages 1745-1755, Minneapolis, Minnesota. Association for Computational Linguistics.

Yinhan Liu, Myle Ott, Naman Goyal, Jingfei Du, Mandar Joshi, Danqi Chen, Omer Levy, Mike Lewis, Luke Zettlemoyer, and Veselin Stoyanov. 2019b. Roberta: A robustly optimized BERT pretraining approach. CoRR, abs/1907.11692.

Ramesh Nallapati, Bowen Zhou, Cicero dos Santos, Çağlar Gulçehre, and Bing Xiang. 2016. Abstractive text summarization using sequence-to-sequence RNNs and beyond. In Proceedings of The 20th SIGNLL Conference on Computational Natural Language Learning, pages 280-290, Berlin, Germany. Association for Computational Linguistics.

Rodrigo Nogueira and Kyunghyun Cho. 2019. Passage re-ranking with BERT. CoRR, abs/1901.04085.

Adam Paszke, Sam Gross, Francisco Massa, Adam Lerer, James Bradbury, Gregory Chanan, Trevor Killeen, Zeming Lin, Natalia Gimelshein, Luca Antiga, Alban Desmaison, Andreas Köpf, Edward Yang, Zachary DeVito, Martin Raison, Alykhan Tejani, Sasank Chilamkurthy, Benoit Steiner, Lu Fang, Junjie Bai, and Soumith Chintala. 2019. Pytorch: An imperative style, high-performance deep learning library. In Advances in Neural Information Processing Systems 32: Annual Conference on Neural Information Processing Systems 2019, NeurIPS 2019, 8-14 December 2019, Vancouver, BC, Canada, pages 8024-8035. 
Romain Paulus, Caiming Xiong, and Richard Socher. 2018. A deep reinforced model for abstractive summarization. In 6th International Conference on Learning Representations, ICLR 2018, Vancouver, BC, Canada, April 30 - May 3, 2018, Conference Track Proceedings. OpenReview.net.

Alec Radford, Jeffrey Wu, Rewon Child, David Luan, Dario Amodei, and Ilya Sutskever. 2018. Language models are unsupervised multitask learners.

Pranav Rajpurkar, Jian Zhang, Konstantin Lopyrev, and Percy Liang. 2016. Squad: 100, 000+ questions for machine comprehension of text. In Proceedings of the 2016 Conference on Empirical Methods in Natural Language Processing, EMNLP 2016, Austin, Texas, USA, November 1-4, 2016, pages 2383-2392. The Association for Computational Linguistics.

Abigail See, Peter J. Liu, and Christopher D. Manning. Get To The Point: Summarization with PointerGenerator Networks. In Proceedings of the 55th Annual Meeting of the Association for Computational Linguistics (Volume 1: Long Papers), pages 10731083. Association for Computational Linguistics.

Josef Steinberger and Karel Jezek. 2004. Using latent semantic analysis in text summarization and summary evaluation.

Sandeep Subramanian, Raymond Li, Jonathan Pilault, and Christopher J. Pal. 2019. On extractive and abstractive neural document summarization with transformer language models. CoRR, abs/1909.03186.

Ming Tu, Kevin Huang, Guangtao Wang, Jing Huang, Xiaodong He, and Bowen Zhou. 2019. Select, answer and explain: Interpretable multi-hop reading comprehension over multiple documents. CoRR, abs/1911.00484.

Ming Tu, Kevin Huang, Guangtao Wang, Jing Huang, Xiaodong He, and Bowen Zhou. 2020. Select, answer and explain: Interpretable multi-hop reading comprehension over multiple documents. national conference on artificial intelligence.

Lucy Vanderwende, Hisami Suzuki, Chris Brockett, and Ani Nenkova. 2007. Beyond sumbasic: Taskfocused summarization with sentence simplification and lexical expansion. Inf. Process. Manag., 43(6):1606-1618.

Ashish Vaswani, Noam Shazeer, Niki Parmar, Jakob Uszkoreit, Llion Jones, Aidan N. Gomez, Lukasz Kaiser, and Illia Polosukhin. 2017. Attention is all you need. In Advances in Neural Information Processing Systems 30: Annual Conference on Neural Information Processing Systems 2017, 4-9 December 2017, Long Beach, CA, USA, pages 5998-6008.

Alex Wang, Amanpreet Singh, Julian Michael, Felix Hill, Omer Levy, and Samuel R. Bowman. 2019. GLUE: A multi-task benchmark and analysis platform for natural language understanding. In International Conference on Learning Representations.
Thomas Wolf, Lysandre Debut, Victor Sanh, Julien Chaumond, Clement Delangue, Anthony Moi, Pierric Cistac, Tim Rault, R'emi Louf, Morgan Funtowicz, and Jamie Brew. 2019. Huggingface's transformers: State-of-the-art natural language processing. ArXiv, abs/1910.03771.

Zhilin Yang, Peng Qi, Saizheng Zhang, Yoshua Bengio, William W. Cohen, Ruslan Salakhutdinov, and Christopher D. Manning. 2018. Hotpotqa: A dataset for diverse, explainable multi-hop question answering. In Proceedings of the 2018 Conference on Empirical Methods in Natural Language Processing, Brussels, Belgium, October 31 - November 4, 2018, pages 2369-2380. Association for Computational Linguistics.

Zichao Yang, Diyi Yang, Chris Dyer, Xiaodong He, Alex Smola, and Eduard Hovy. 2016. Hierarchical attention networks for document classification. In Proceedings of the 2016 Conference of the North American Chapter of the Association for Computational Linguistics: Human Language Technologies, pages 1480-1489, San Diego, California. Association for Computational Linguistics.

Manzil Zaheer, Guru Guruganesh, Avinava Dubey, Joshua Ainslie, Chris Alberti, Santiago Ontanon, Philip Pham, Anirudh Ravula, Qifan Wang, Li Yang, and Amr Ahmed. 2020. Big bird: Transformers for longer sequences.

Rowan Zellers, Yonatan Bisk, Roy Schwartz, and Yejin Choi. 2018. SWAG: A large-scale adversarial dataset for grounded commonsense inference. CoRR, abs/1808.05326.

Jingqing Zhang, Yao Zhao, Mohammad Saleh, and Peter J. Liu. 2019a. Pegasus: Pre-training with extracted gap-sentences for abstractive summarization.

Xingxing Zhang, Mirella Lapata, Furu Wei, and Ming Zhou. 2018. Neural latent extractive document summarization. In Proceedings of the 2018 Conference on Empirical Methods in Natural Language Processing, pages 779-784, Brussels, Belgium. Association for Computational Linguistics.

Xingxing Zhang, Furu Wei, and Ming Zhou. 2019b. HIBERT: document level pre-training of hierarchical bidirectional transformers for document summarization. In Proceedings of the 57th Conference of the Association for Computational Linguistics, ACL 2019, Florence, Italy, July 28-August 2, 2019, Volume 1: Long Papers, pages 5059-5069. Association for Computational Linguistics.

Chen Zhao, Chenyan Xiong, Corby Rosset, Xia Song, Paul N. Bennett, and Saurabh Tiwary. 2020. Transformer-xh: Multi-evidence reasoning with extra hop attention. In 8th International Conference on Learning Representations, ICLR 2020, Addis Ababa, Ethiopia, April 26-30, 2020. OpenReview.net. 
Ming Zhong, Pengfei Liu, Yiran Chen, Danqing Wang, Xipeng Qiu, and Xuanjing Huang. 2020. Extractive summarization as text matching. In Proceedings of the 58th Annual Meeting of the Association for Computational Linguistics, pages 6197-6208, Online. Association for Computational Linguistics.

Ming Zhong, Pengfei Liu, Danqing Wang, Xipeng Qiu, and Xuanjing Huang. 2019. Searching for effective neural extractive summarization: What works and what's next. In Proceedings of the 57th Conference of the Association for Computational Linguistics, ACL 2019, Florence, Italy, July 28-August 2, 2019, Volume 1: Long Papers, pages 1049-1058. Association for Computational Linguistics.

Qingyu Zhou, Nan Yang, Furu Wei, Shaohan Huang, Ming Zhou, and Tiejun Zhao. 2018. Neural document summarization by jointly learning to score and select sentences. In Proceedings of the 56th Annual Meeting of the Association for Computational Linguistics (Volume 1: Long Papers), pages 654663, Melbourne, Australia. Association for Computational Linguistics.

Yukun Zhu, Ryan Kiros, Richard S. Zemel, Ruslan Salakhutdinov, Raquel Urtasun, Antonio Torralba, and Sanja Fidler. 2015. Aligning books and movies: Towards story-like visual explanations by watching movies and reading books. In 2015 IEEE International Conference on Computer Vision, ICCV 2015 , Santiago, Chile, December 7-13, 2015, pages 19-27. IEEE Computer Society. 


\section{A Baselines: Implementation Details}

BERTSUMEXT: For all experiments with BERTSUMEXT, we started with the original implementation ${ }^{3}$ and adapted the code to build the sliding windows version. This implementation leverage bert-base-uncased pre-trained model and its associated hyperparameters. We use windows of width 800 with an overlap of 300 tokens between two following windows. If a sentence is in multiple windows, we select its [CLS] representation in the window that maximizes the number of surrounding tokens. We finetune the model for 5 epochs using Adam optimizer with an initial learning rate of $1 \times 10^{-5}, \beta_{1}=0.9, \beta_{2}=0.999$.

Longformer-Ext: We built the Longformer-Ext baseline from the Longformer implementation released by HuggingFace ${ }^{4}$. We use the official longformer-base-4096 pre-trained model trained by AllenAI ${ }^{5}$. This model is based on RoBERTabase and its associated hyperparameters. To increase the maximal position embedding, we drop the pre-trained positional embedding parameters and train a novel token embedding layer to scale Longformer-Ext input up to 12294 tokens. This model computes a sliding self-attention with a window size of 512 tokens on all its 12 Transformer layers. We finetune the model for 5 epochs with only local attention because of memory constraints, using Adam optimizer with an initial learning rate of $1 \times 10^{-5}, \beta_{1}=0.9$, $\beta_{2}=0.999$, no learning rate warmup and a linear decay of the learning rate.

Reformer-Ext: We started from the HuggingFace implementation of Reformer to build Reformer-Ext baseline. We use a Reformer configuration composed of six layers of attention. We use Locality-Sensitive Hashing Attention with 128 buckets on the input sequence and Local Selfattention on chunks of 64 tokens. We use hidden sates of dimension 256, a feed-forward layer of dimension 512, and 12 attention heads in Transformer encoders. We train this model for 5 epochs using Adam optimizer with an initial learning rate of $1 \times 10^{-5}, \beta_{1}=0.9, \beta_{2}=0.999$, no learning rate warmup and a linear decay of the

\footnotetext{
${ }^{3}$ https://github.com/nlpyang/PreSumm

${ }^{4}$ https://github. com/huggingface/ transformers

${ }^{5}$ https: //github.com/allenai/longformer
}

learning rate.

\section{B Datasets Statistics}

Figure 4 presents the distribution of the document lengths in arXiv, PubMed and CNN/DailyMail, after tokenization with pretrained BERT-base tokenizer. It also provides the histograms of the position of the [CLS] tokens of the Oracle sentences in input documents. One can see that the three datasets contain an important number of documents longer than 512 tokens, the standard length limitation of pre-trained language models. However, one can also notice that CNN/DailyMail contains a large part of its Oracle sentences within this first window of 512 tokens. As a consequence, a model that is not able to "read" beyond this limitation is not penalized. It is also a reason why Lead baseline is quite strong on this dataset. On the contrary, on arXiv and PubMed, one can see that a large part of Oracle sentences occur beyond this 512 windows. This explains why models capable of reading long sequences are required to achieve good results on these datasets. 
ArXiv
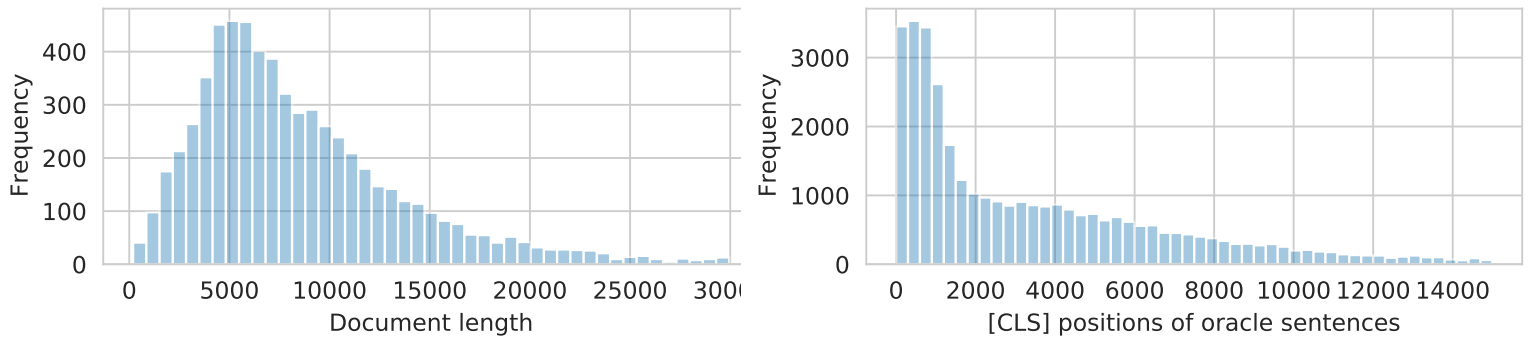

\section{PubMed}
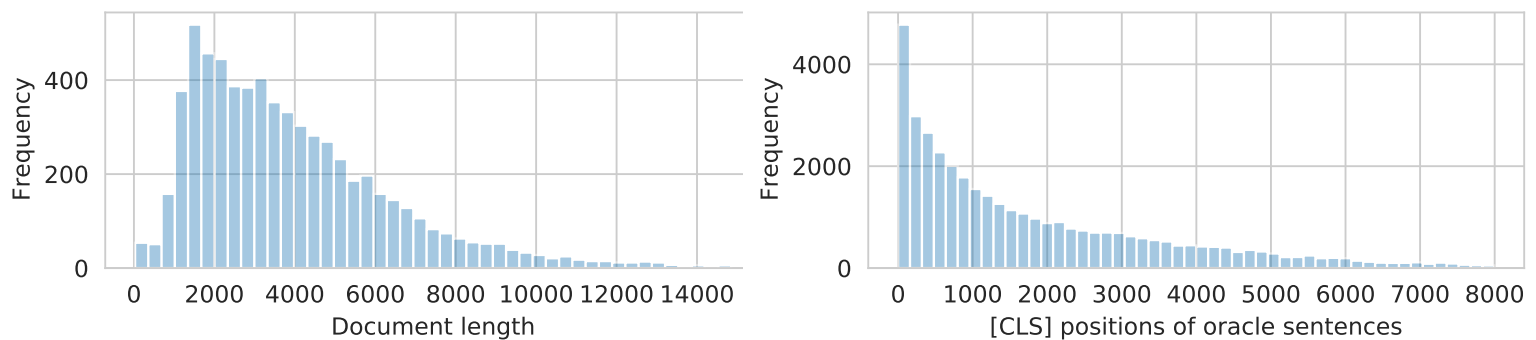

CNN/DailyMail
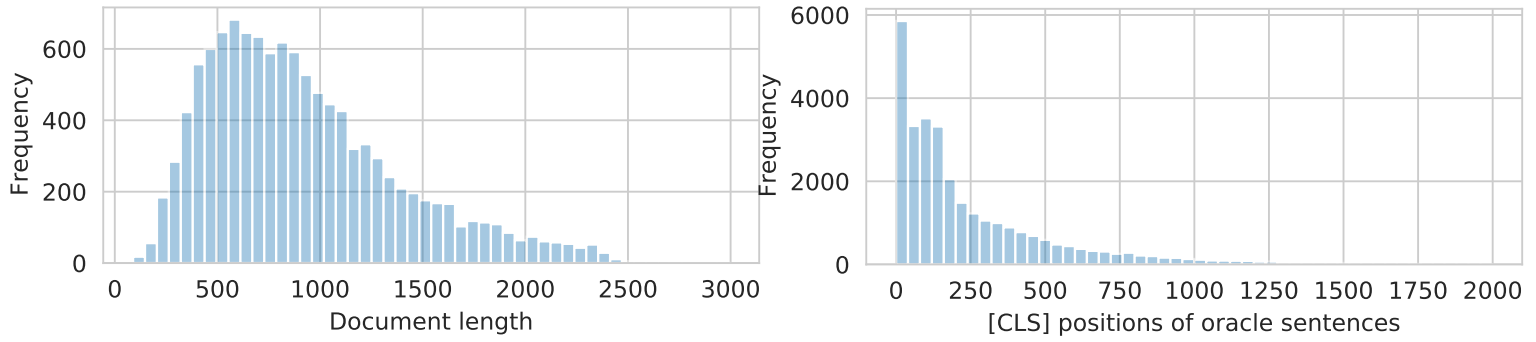

Figure 4: Document lengths after tokenization with pretrained BERT-base tokenizer and position of the [CLS] tokens of Oracle sentences in the input documents. 


\section{PubMed Summaries}

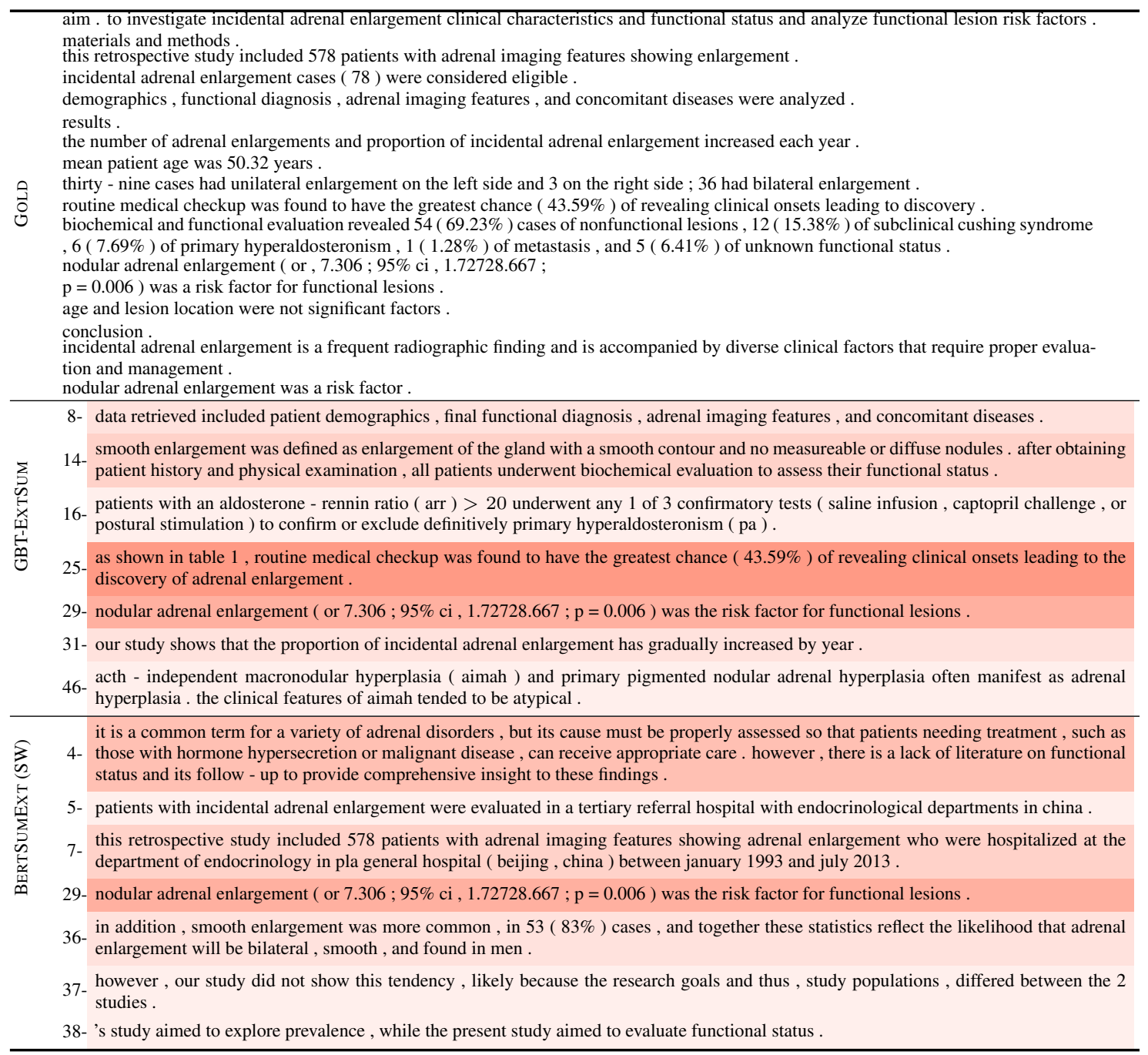




\begin{tabular}{|c|c|}
\hline \multicolumn{2}{|r|}{$\begin{array}{l}\text { background and objective } \\
\text { antimicrobial resistance is now a major challenge to clinicians for treating patients . } \\
\text { hence, this short term study was undertaken to detect the incidence of multidrug - resistant ( mdr ), extensively drug - resistant ( xdr ), } \\
\text { and pandrug - resistant ( pdr ) bacterial isolates in a tertiary care hospital . } \\
\text { material and methods . } \\
\text { the clinical samples were cultured and bacterial strains were identified in the department of microbiology . } \\
\text { the antibiotic susceptibility profile of different bacterial isolates was studied to detect mdr, xdr, and pdr bacteria . } \\
\text { results . the antibiotic susceptibility profile of } 1060 \text { bacterial strains was studied . } \\
393(37.1 \%) \text { bacterial strains were mdr, } 146(13.8 \%) \text { strains were xdr, and no pdr was isolated . } \\
\text { all ( } 100 \% \text { ) gram negative bacterial strains were sensitive to colistin whereas all ( } 100 \% \text { ) gram positive bacterial strains were sensitive to } \\
\text { vancomycin. } \\
\text { conclusion. } \\
\text { close monitoring of mdr, xdr, or even pdr must be done by all clinical microbiology laboratories to implement effective measures to reduce } \\
\text { the menace of antimicrobial resistance. }\end{array}$} \\
\hline & $\begin{array}{l}\text { multidrug resistant ( } \mathrm{mdr} \text { ) was defined as acquired nonsusceptibility to at least one agent in three or more antimicrobial categories . } \\
\text { extensively drug }\end{array}$ \\
\hline 36- & no mdr or xdr strain was isolated from streptococcus sp. all ( $100 \%$ ) gram positive cocci were sensitive to vancomycin and linezolid . \\
\hline 38- & e. coli was the commonest isolate 261 ( $35 \%$ ), followed by pseudomonas aeruginosa $212(28.4 \%)$. \\
\hline 40- & $\begin{array}{l}\text { out of } 200 \text { klebsiella pneumoniae strains isolated, } 75(37.5 \%) \text { and } 25(12.5 \%) \text { were detected as mdr and xdr, respectively . out of } 42 \\
\text { acinetobacter and other nonfermenter species isolated , } 19(45.2 \%) \text { and } 8(19 \%) \text { were mdr and xdr strains, respectively. amongst } 250 \\
\text { gnb - mdr strains isolated, }\end{array}$ \\
\hline & , it has been reported that most frequent mdr pathogens were pseudomonas aeruginosa followed by e. coli . \\
\hline & $\begin{array}{l}\text { unless and until multidrug resistant organisms are detected and their incidence is known, the strategies for their control can not be adopted } \\
\text { properly in healthcare setup. hence, detection, prevention of transmission of mdros by following infection control practices, antimicrobial } \\
\text { surveillance, and stewardship are need of the hour. }\end{array}$ \\
\hline 69- & $\begin{array}{l}\text { we hereby conclude that early detection and close monitoring of mdr, } \mathrm{xdr} \text {, or even pdr bacterial strains must be started by all clinical } \\
\text { microbiology laboratories to reduce the menace of antimicrobial resistance which is now a global problem. }\end{array}$ \\
\hline 9- & $\begin{array}{l}\text { this short term cross - sectional study was conducted in the department of microbiology from } 15 \text { th of april to } 15 \text { th of july , } 2014 \text {. } \\
\text { the bacterial strains were isolated from different clinical samples and were identified by conventional methods . }\end{array}$ \\
\hline & $\begin{array}{l}\text { methicillin resistant staphylococcus aureus ( mrsa ) strains were detected by meca - mediated oxacillin resistance using cefoxitin disk ( } 30 \\
\mathrm{~g} \text { ) on mueller hinton ( } \mathrm{mh} \text { ) agar plate inoculated with test strains as per standard disk diffusion recommendations and incubated at } 3335 \mathrm{c} \\
\text { for } 1618 \text { hours. }\end{array}$ \\
\hline 20 & $\begin{array}{l}\text { an increase in diameter of } 5 \mathrm{~mm} \text { with ceftazidime plus clavulanic acid as compared to ceftazidime disk alone was considered positive for } \\
\text { esbl detection. }\end{array}$ \\
\hline 36- & no mdr or xdr strain was isolated from streptococcus sp. all ( $100 \%$ ) gram positive cocci were sensitive to vancomycin and linezolid . \\
\hline & e. coli was the commonest isolate 261 ( $35 \%$ ), followed by pseudomonas aeruginosa $212(28.4 \%)$. \\
\hline & $\begin{array}{l}\text { the limitation of this study is that this is a single center study for only three - month period in a tertiary care hospital in central india . to } \\
\text { reflect the trend of infections caused by mdr and xdr strains of bacteria in the region, a multicenter study involving all types of healthcare } \\
\text { setups for a minimum period of one year }\end{array}$ \\
\hline
\end{tabular}


background suicide is a grave public health issue that is responsible for a high mortality rate among individuals aged 1544 years . attitudes toward suicide among medical staff members have been associated with appropriate therapeutic responses to suicidal individuals the aim of this study was to examine the effects of parental rearing on attitudes toward suicide among japanese medical college students.methodswe examined the association between parental bonding and attitudes toward suicide in 160 medical college students in japan

the parental bonding instrument was used to assess the attitudes and behaviors of parents .

the attitudes toward suicide were evaluated using the japanese version of the attitudes toward suicide questionnaire.resultsthe mean age of

the subjects was 25.24 .0 years old

8 the majority of the participants in our study agreed that anyone could commit suicide ( $88.8 \%$ ) and that suicide is preventable ( $86.3 \%$ ) after adjusting for age and sex , multivariate regression analysis revealed that maternal care approached a statistically significant association with the right to suicide attitude . under the same conditions ,

maternal care was shown to be significantly associated with the common occurrence attitude

no other significant relationships were observed between parental bonding and attitudes toward suicide.conclusionthis study suggests that

a higher level of maternal care ensures that children think that suicide occurs less commonly .

the promotion of best practices for suicide prevention among medical students is needed.

child rearing support might be associated with suicide prevention .

previous studies have shown that difficulties with parental bonding during childhood could be a predisposing factor for the onset of many

3- psychiatric conditions, such as anxiety , depressive states , and maladjusted behaviors. 68 parental bonding and premorbid personality traits play an important role in shaping the developmental trajectory of an individual, including his / her ability to adjust to stressful events .

5- the objective of this study was to investigate whether parental bonding is associated with attitudes toward suicide among medical college students in japan.

8- the demographic data ( age and sex ) were obtained from self - questionnaires and interviews .

14- higher scores on the care and protection dimensions reveal that participants perceive their parents to be more caring and/or protective .

39- right to suicide was significantly associated with common occurrence, unjustified behavior, and preventability / readiness to help .

43- the majority of the participants in our study agreed that anyone could commit suicide ( $88.8 \%$ ) and that suicide is preventable ( $86.3 \%$ ).

44 in addition, the multiple regression analysis revealed that participants who reported a higher level of maternal care thought that suicide was a common occurrence and tended to think that people do not have the right to commit suicide .

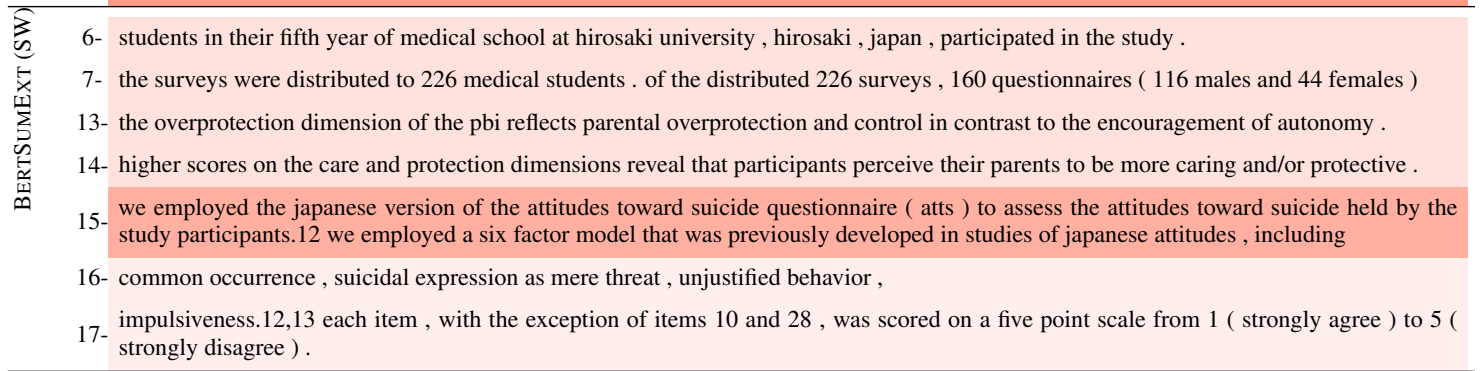




\section{ArXiv Summaries}

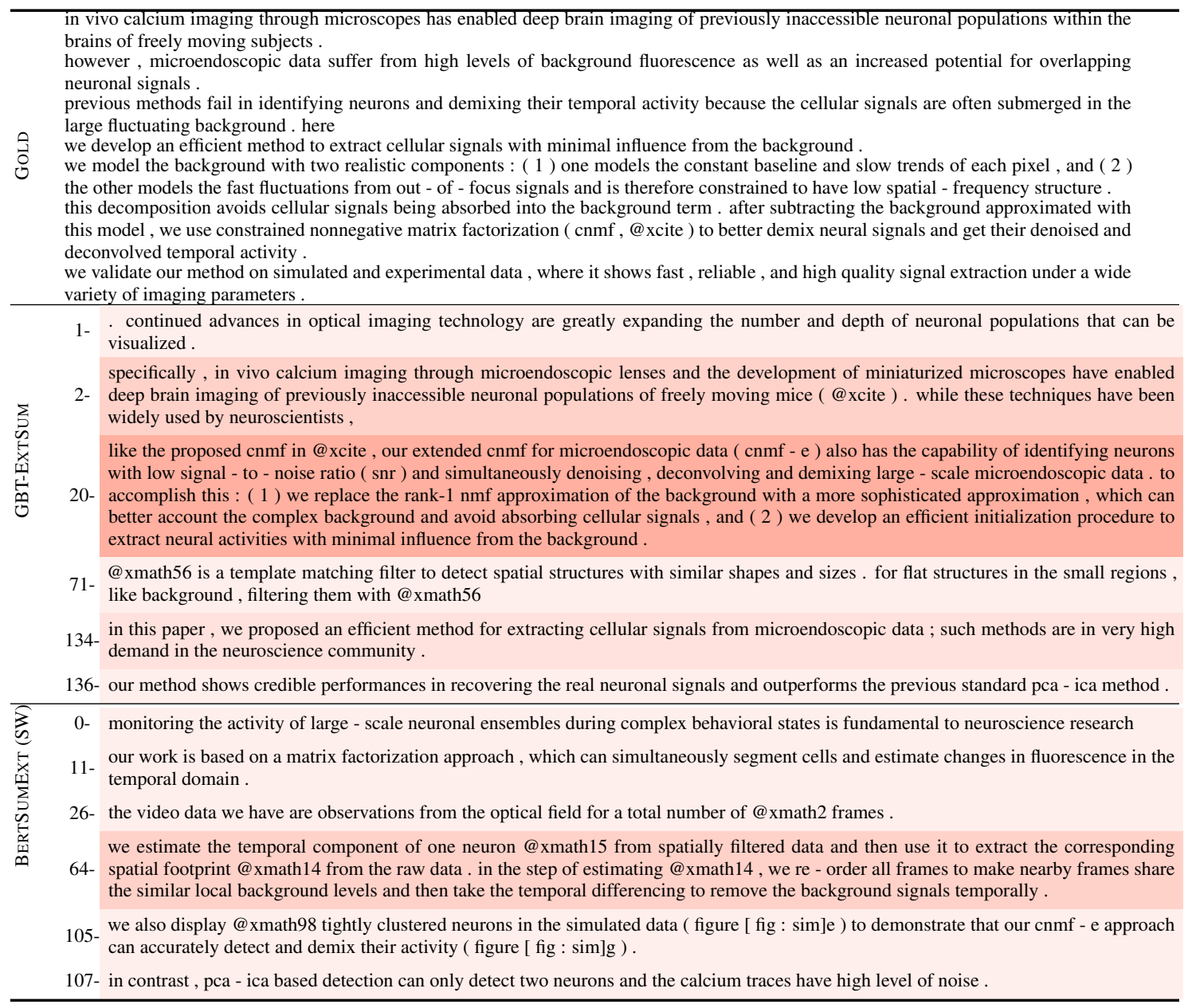


the fundamental quantity of interest is the sample complexity : the number of samples required to learn to a specified level of accuracy . here we consider learning over the set of all computable labeling functions

since the vc - dimension is infinite and a priori ( uniform ) bounds on the number of samples are impossible, we let the learning algorithm decide when it has seen sufficient samples to have learned . we first show that learning in this setting is indeed possible, and develop a learning algorithm .

we then show, however, that bounding sample complexity independently of the distribution is impossible .

notably, this impossibility is entirely due to the requirement that the learning algorithm be computable, and not due to the statistical nature of the problem

6- an alternative approach, and one we follow in this paper, is simply to consider a single learning model that includes all possible classification methods.

8- since the vc - dimension is clearly infinite, there are no uniform bounds ( independent of the distribution and the target concept ) on the 8- number of samples needed to learn accurately @xcite

, it is natural to allow the learning algorithm to decide when it has seen sufficiently many labeled samples based on the training samples

10- seen up to now and their labels . since the above learning model includes any practical classification scheme, we term it universal ( pac- ) learning .

11- we first show that there is a computable learning algorithm in our universal setting .

9. our results imply that computable learning algorithms in the universal setting must waste samples " in the sense of requiring more samples than is necessary for statistical reasons alone.

81- then we will contrast this to the case of an uncomputable learning algorithm .

( semantic requirements ) for any @xmath27, for any concept @xmath8, and distribution @xmath9 over @xmath2, if the oracle returns 50-pairs@xmath28 for @xmath29 drawn iid from @xmath9, then @xmath0 always halts , and with probability at least @xmath12 outputs a 50- hypothesis@xmath13 such that @xmath30i\{ varepsilon\}\$ ]

64- suppose@ @xmath36 is an infinite sequence of iid samples drawn from @ xmath9.

75- the learning algorithm queries the oracle as necessary for new learning samples and their labeling

78- note that it seems necessary to expand the hypothesis space to include all partial recursive functions because the concept space of total 78- recursive functions does not have a recursive enumeration (it is uncomputable whether a given program is total recursive or not ).

79- we will see in theorem [ thm : nobound ] that there is no bound @ xmath55 on the number of samples queried by any computable learning algorithm in our setting .

80- let us obtain some intuition for why that is true for the above learning algorithm .

in this paper, we propose majority voting neural networks for sparse signal recovery in binary compressed sensing

the majority voting neural network is composed of several independently trained feedforward neural networks employing the sigmoid function as an activation function

our empirical study shows that a choice of a loss function used in training processes for the network is of prime importance

we found a loss function suitable for sparse signal recovery , which includes a cross entropy - like term and an @ xmath0 regularized term from the experimental results

, we observed that the majority voting neural network achieves excellent recovery performance, which is approaching the optimal performance as the number of component nets grows.

the simple architecture of the majority voting neural networks would be beneficial for both software and hardware implementations

5 40- requires only several matrix - vector products to obtain an output signal, which is an estimate signal of the sparse vector @xmath12.

s the signal propagates from left to right and the output signal @ xmath17 eventually comes out from the output layer . the network should 48- be trained so that the output signal @xmath17 is an accurate estimation of the original sparse signal @ xmath12.

168- in this paper, we proposed sparse signal recovery schemes based on neural networks for binary compressed sensing .

69- our empirical study shows a choice of the loss function used for training neural networks is of prime importance to achieve excellent reconstruction performance.

170- we found a loss function suitable for this purpose, which includes a cross entropy like term and an @ xmath0 regularized term .

173- the simple architecture of the majority voting neural network would be beneficial for both software and hardware implementation .

19- the paper @xcite presents binary iterative hard thresholding ( biht ) algorithm by reforming iterative hard thresholding ( iht ) algorithm

20- although the known sparse recovery algorithms exhibit reasonable sparse recovery performance, it may not be suitable for applications in 20- high speed wireless communications .

48 the signal propagates from left to right and the output signal@xmath17 eventually comes out from the output layer . the network should be trained so that the output signal @xmath17 is an accurate estimation of the original sparse signal @xmath12.

the outputs from these neural network are combined by soft majority voting nodes and the final estimation vector is obtained by rounding

the output from the soft majority voting nodes . combining a several neural networks to obtain improved performance is not a novel idea e.g. , @xcite , but it will be shown that the idea is very effective for our purpose . from statistics of reconstruction errors occurred in our computer experiments, we observed that many reconstruction error events ( i.e. , @ xmath97 ) occur due to only one symbol mismatch

149- note that implementation of neural networks with fpga is recently becoming a hot research topic @ xcite .

the length of the sparse signal is set to @xmath59 and the sparseness parameter is set to @xmath110 . ), width=317 ] from fig.[fig

151- : rr_and_m_k6 ], we can observe significant improvement in recovery performance compared with the performance of the single neural network . a single feedforward neural network discussed in the previous section 